\title{
Infrainguinal occlusions: the real challenge for the endovascular specialist
}

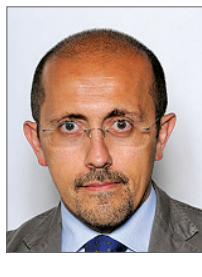

\author{
Eugenio Stabile $^{1 *}$, MD, PhD; Tullio Tesorio ${ }^{2}, \mathrm{MD}$; Giovanni Esposito ${ }^{1}, \mathrm{MD}$ \\ 1. Division of Cardiology, Department of Advanced Biomedical Sciences, Federico II University, Naples, Italy; \\ 2. Clinica Montevergine, Mercogliano, Italy
}

Endovascular treatment of chronic total occlusions (CTO) of the infrainguinal artery is a reliable and safe alternative therapeutic option to surgery for the treatment of obstructive disease.

Thanks to growing experience and the use of drug-eluting technologies, the long-term results of de novo ${ }^{1}$ and recurrent occlusions $^{2}$ are satisfactory. Therefore, at present, the remaining limiting factor is gaining the true lumen distal to the occlusion (i.e., procedural success).

Today, the ability of each endovascular specialist to gain the distal lumen of an infrainguinal CTO is improving more and more $^{3}$. At the same time, it is becoming more frequent than ever to have to face these extremely challenging occlusions in complex patients with difficult anatomic settings.

In the last decade, there has been a proliferation of strategies and devices designed to improve procedural success and outcomes in patients with infrainguinal CTO. Dedicated wires, support microcatheters, pedal access, intrastent punctures, re-entry devices and centre crossing devices have recently been developed ${ }^{4}$.

There are two possible procedural strategies to address an infrainguinal occlusion (Figure 1).

Subintimal navigation: a well-characterised, historical technique, which limits the choice of adjunctive devices and often requires the use of re-entry devices (i.e., angio-guided, IVUSguided, OCT-guided) to achieve the true distal lumen positioning of the guidewire.

Central lumen navigation: clinically preferred on account of the fact that it maximises therapeutic options allowing the use of all adjunctive devices. Often, dedicated devices (i.e., basket-like, centring balloons) designed to facilitate central lumen guidewire navigation are needed.

The most challenging of the endovascular therapeutic procedures remains the CTO located at the popliteal and infrapopliteal level. Not only do these procedures require significant time, radiation and contrast use, but also they are associated with suboptimal procedural success and higher complication (i.e., arterial dissection and/or perforation, distal embolisation and bleeding) rates.

In this issue, Jalal et $\mathrm{al}^{5}$ report the results of a single-centre trial evaluating the safety and efficacy of two different devices for central Article, see page 1063

lumen recanalisation of peripheral CTO lesions after an unsuccessful attempt with a conventional technique. Despite a relatively long average lesion length $(>15 \mathrm{~cm})$, the procedural success was elevated and achieved with a relatively short crossing time ( $<6$ minutes), low volume of contrast medium and short fluoroscopy time, and without paying the price of a higher procedural complication rate.

These favourable outcomes were achieved despite the fact that the vast majority of treated lesions were popliteal or infrapopliteal, as opposed to most other studies where the majority of treated CTO were superficial femoral artery lesions.

As operators, we should always remember that recanalisation of popliteal and infrapopliteal occlusions is associated with unsuccessful results and various potential complications (arterial perforation, collateral branch occlusion), mainly related to the use of subintimal recanalisation. The devices tested in this study allow 


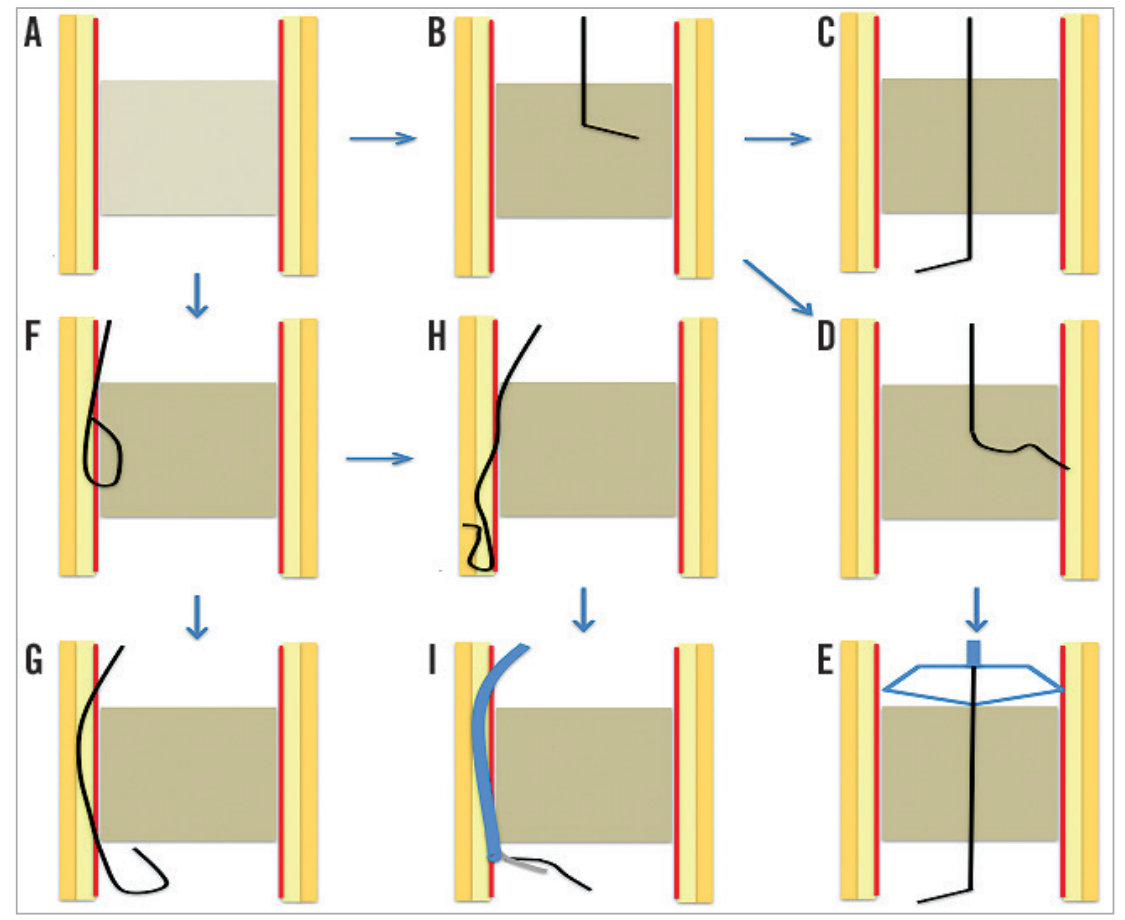

Figure 1. Strategies for endovascular recanalisation of peripheral artery atherothrombotic occlusion. A) Schematic representation of a peripheral artery atherothrombotic occlusion. The arterial artery is represented as composed of three layers (intima: red; media: pale yellow; adventitia: dark yellow). An atherothrombotic plaque (grey) is completely obstructing the arterial lumen. B) Central lumen navigation strategy. A dedicated guidewire is advanced and navigated into the true lumen of the atherothrombotic occlusion. C) Successful recanalisation of the atherothrombotic occlusion using a central lumen strategy. The wire tip is located in the true lumen, distal to the atherothrombotic occlusion. D) Unsuccessful recanalisation of the atherothrombotic occlusion using a central lumen strategy. The wire cannot be advanced into the central lumen, bends and deviates to the vessel wall. E) Successful recanalisation of the atherothrombotic occlusion using a central lumen strategy thanks to the use of a dedicated centring device able to support the wire navigation in the central lumen. F) Subintimal navigation strategy. A dedicated wire has been advanced and looped in the subintimal space of the atherothrombotic occlusion. G) Successful recanalisation of the atherothrombotic occlusion using a subintimal navigation strategy. The looped tip of the wire is advanced into the subintimal space distal to the occluded arterial segment and successfully re-entered into the true lumen, distal to the atherothrombotic occlusion. H) Unsuccessful recanalisation of the atherothrombotic occlusion after a subintimal navigation strategy. The looped tip of the wire is advanced into the subintimal space distal to the occluded arterial segment but re-entry in the true lumen distal to the atherothrombotic occlusion failed. I) Successful recanalisation of the atherothrombotic occlusion with a subintimal navigation strategy using a dedicated re-entry catheter. The dedicated catheter is advanced into the subintimal space distal to the atherothrombotic occlusion and allows the distal tip of the guidewire to re-enter into the true arterial lumen.

obtaining a central lumen recanalisation and maximise therapeutic options, including the use of directional atherectomy to treat calcific obstructions ${ }^{6}$.

There are some current economic and procedural concerns about the broad application of crossing devices in all popliteal and infrapopliteal lesions.

- It increases the cost of the procedure considerably.

- The prolonged procedural time, as a result of crossing attempts with different strategies (intraluminal, subintimal, centring devices) and the necessity of repeated angiograms, can restrict the application of these devices in elderly fragile patients or in patients with chronic kidney disease.

There is no current literature describing the value of using central lumen devices as a first-line attempt to recanalise infrapopliteal arteries. It would be desirable to have a study investigating whether this approach is able to reduce the overall procedural time, costs and contrast load. The use of dedicated (re-entry or central lumen) devices allows increasing procedural success, and more data on their role in facilitating complex procedures are needed.

One final comment should be made on our role as physicians involved in the research and cure of peripheral athero-occlusive disease. We should praise the efforts of industry and physicians in developing and validating new devices with the aim of facilitating our work as endovascular specialists, but we should never forget to continue our efforts in spreading awareness of atheroocclusive peripheral artery disease.

Infrainguinal occlusions are more frequent in patients with a long history of untreated peripheral artery disease and, today, we need tools which will facilitate achieving a satisfactory clinical success rate. Our ambition should be to reach a point where we 
will approach these obstructions at a very early stage after symptom onset, when they can be treated easily with high procedural success and low complication rates.

\section{Conflict of interest statement}

The authors have no conflicts of interest to declare.

\section{References}

1. Laird JR, Schneider PA, Tepe G, Brodmann M, Zeller T, Metzger C, Krishnan P, Scheinert D, Micari A, Cohen DJ, Wang H, Hasenbank MS, Jaff MR; IN.PACT SFA Trial Investigators. Durability of Treatment Effect Using a Drug-Coated Balloon for Femoropopliteal Lesions: 24-Month Results of IN.PACT SFA. $J$ Am Coll Cardiol. 2015; 66:2329-38.

2. Virga V, Stabile E, Biamino G, Salemme L, Cioppa A, Giugliano G, Tesorio T, Cota L, Popusoi G, Pucciarelli A, Esposito G, Trimarco B, Rubino P. Drug-eluting balloons for the treatment of the superficial femoral artery in-stent restenosis: 2-year follow-up. JACC Cardiovasc Interv. 2014;7:411-5.

3. Maertens H, Aggarwal R, Macdonald S, Vermassen F, Van Herzeele I; FOundER group. Transatlantic Multispecialty Consensus on Fundamental Endovascular Skills: Results of a Delphi Consensus Study. Eur J Vasc Endovasc Surg. 2015 Oct 9. [Epub ahead of print].

4. Lorenzoni R, Ferraresi R, Manzi M, Roffi M. Guidewires for lower extremity artery angioplasty: a review. EuroIntervention. 2015;11:799-807.

5. Jalal S, Lalonde TA, Yamasaki H, Boshara A, Rosman HS, Mehta RH, Davis TP. Novel CentreCross and MultiCross devices for the treatment of infrainguinal chronic total occlusions: initial single-centre experience. EuroIntervention. 2016;11:1063-9.

6. Cioppa A, Stabile E, Tesorio T. Commentary: Never Forget Your Old Toys When You Get New Ones. J Endovasc Ther. 2015;22:853-4. 